\title{
Pseudo-Tetrahedral Polyhalocubanes: Synthesis, Structures, and Parity Violating Energy Differences
}

Andrey A. Fokin, ${ }^{\star a, b}$ Peter R. Schreiner, ${ }^{* a}$ Robert Berger, ${ }^{c}$ Gregory H. Robinson, ${ }^{d}$ Pinrong Wei, ${ }^{d}$ Charles F. Campana ${ }^{e}$

${ }^{a}$ Institut für Organische Chemie der Justus-Liebig-Universität, Heinrich-Buff-Ring 58, 35392 Giessen, Germany; ${ }^{b}$ Department of Organic Chemistry, Kiev Polytechnic Institute, pr. Pobedy, 37, 03056 Kiev, Ukraine; ${ }^{\circ}$ Frankfurt Institute for Advanced Studies, J. W. Goethe University, Max-von-Laue-Str. 1, 60438 Frankfurt, Germany; ${ }^{d}$ Department of Chemistry, University of Georgia, Athens, GA, 30602-2556, USA; ${ }^{e}$ Advanced X-ray Solutions, Bruker AXS Inc., Madison, WI 12345, USA; Andrey.Fokin@org.chemie.uni-giessen.de,prs@org.chemie.uni-giessen.de

\section{Supplementary Information}

\section{Experimental details}

\subsection{Experimental procedures for the preparation of halocubanes}

${ }^{1} \mathrm{H}$ and ${ }^{13} \mathrm{C}$ NMR spectra were recorded on a Bruker AM 400 spectrometer at $298 \mathrm{~K}$ in 5 mm NMR tubes, operating at 400.1 and $100.6 \mathrm{MHz}$, respectively. The chemical shifts ( $\delta$ values) were obtained in $\mathrm{CDCl}_{3}$ solutions unless otherwise noted and referenced to residual $\mathrm{CHCl}_{3}\left(\delta{ }^{1} \mathrm{H}\right.$ $7.25 \mathrm{ppm})$ and $\left({ }^{13} \mathrm{C} 77.2 \mathrm{ppm}\right)$. The ${ }^{13} \mathrm{C}$ NMR spectra were acquired using the broadband proton decoupling mode and the DEPT technique. The assignments of the ${ }^{13} \mathrm{C}$ resonances of all compounds were deduced from the application of heteronuclear correlation technique and the DEPT technique.

1-Chloro-3-bromo-5-fluorocubane (1). A solution of $60 \mathrm{mg}(0.38 \mathrm{mmol})$ of 1-chloro-3fluoro cubane $(8), 400 \mathrm{mg}(1.21 \mathrm{mmol})$ of $\mathrm{CBr}_{4}, 200 \mathrm{mg}$ of $t-\mathrm{BuOBr}(1.31 \mathrm{mmol})$ in $8 \mathrm{~mL}$ of $\mathrm{CF}_{2} \mathrm{Br}_{2}$ was irradiated for $20 \mathrm{~min}$ at $0{ }^{\circ} \mathrm{C}$ with a $150 \mathrm{~W}$ high-pressure $\mathrm{Hg}$-lamp. The reaction mixture was quenched with a saturated solution of $\mathrm{NaHSO}_{3}$, separated, extracted with $\mathrm{CHCl}_{3}$ $(3 \times 5 \mathrm{~mL})$, the combined organic extracts were evaporated, and the residue was separated by column chromatography $\left(\mathrm{SiO}_{2} /\right.$ pentane) to give $21 \mathrm{mg}$ (23\%) of 1-chloro-3-bromo-5-fluoro cubane (1) as a white solid, m.p. $=73-74{ }^{\circ} \mathrm{C} .{ }^{1} \mathrm{H}$ NMR: $4.68(\mathrm{~m}, 1 \mathrm{H}), 4.48(\mathrm{~m}, 2 \mathrm{H}), 4.18(\mathrm{~m}$, 1H), 3.92 (m, 1H). ${ }^{13} \mathrm{C}$ NMR: 94.5 (d, $\left.J=329 \mathrm{~Hz}, \mathrm{C}\right), 72.9$ (d, $\left.J=24 \mathrm{~Hz}, \mathrm{CH}\right), 65.7$ (d, $J=24$ 
$\mathrm{Hz}, \mathrm{CH}), 65.5(\mathrm{~d}, J=14 \mathrm{~Hz}, \mathrm{CH}), 65.2(\mathrm{~d}, J=24 \mathrm{~Hz}, \mathrm{CH}), 61.5(\mathrm{~d}, J=7 \mathrm{~Hz}, \mathrm{C}), 48.9(\mathrm{~d}, J=8$ Hz, C), 32.8 (d, $J=5$ Hz, CH). MS (M/z): 234, 232, 210, 208, 200, 198, 192, 190, 176, 174, $166,164,156,155,154,135,130,121,120$ (100\%), 119, 111, 109, 99, 95, 94, 87, 85, 81, 76, 75, 74, 70, 69, 61, 60, 62, 57, 51, 50, 49, 39, 38, 37.

1-Chloro-3-bromo-5-iodocubane (2). A solution of $50 \mathrm{mg}(0.23 \mathrm{mmol})$ of 1-bromo-3chloro cubane $(6), 15 \mathrm{mg}(0.06 \mathrm{mmol})$ of iron(II) acetyl acetonate, $10 \mathrm{mg}(0.04 \mathrm{mmol})$ of dibenzoyl peroxide, $0.2 \mathrm{~mL}$ of HOAc in $3 \mathrm{~mL}$ of $n-\mathrm{C}_{6} \mathrm{~F}_{13} \mathrm{I}$ was heated for $48 \mathrm{~h}$ at $100{ }^{\circ} \mathrm{C}$ under argon. Workup of the reaction mixture as above and column chromatography $\left(\mathrm{SiO}_{2} /\right.$ pentane) gave $24 \mathrm{mg}(0.07 \mathrm{mmol}, 30 \%)$ of 1-chloro-3-bromo-5-iodocubane $(\mathbf{2})$ as a white solid, $\mathrm{m} . \mathrm{p} .=$ 145-146 ${ }^{\circ} \mathrm{C} .{ }^{1} \mathrm{H}$ NMR: $4.60(\mathrm{~m}, 1 \mathrm{H}), 4.49$ (m, 1H), $4.41(\mathrm{~m}, 2 \mathrm{H}), 4.25(\mathrm{~m}, 1 \mathrm{H}) .{ }^{13} \mathrm{C}$ NMR: 72.0 $(\mathrm{CH}), 66.9(\mathrm{C}), 65.3(\mathrm{CH}), 65.0(\mathrm{CH}), 64.8(\mathrm{CH}), 54.1(\mathrm{C}), 41.4(\mathrm{CH}), 21.4(\mathrm{C}) . \mathrm{MS}(\mathrm{M} / z)$ : 344, $342,307,284,282,262,238,228,212,180,182,155,138,136$ (100 \%), 111, 101, 75, 74, 62, 50 .

1-Bromo-3-fluoro-5-iodocubane (3). A solution of $100 \mathrm{mg}(0.50 \mathrm{mmol})$ of 1-bromo-3fluoro cubane $(9), 25 \mathrm{mg}(0.1 \mathrm{mmol})$ of iron(II) acetyl acetonate, $15 \mathrm{mg}(0.06 \mathrm{mmol})$ of dibenzoyl peroxide, $0.4 \mathrm{~mL}$ of HOAc in $4.5 \mathrm{~mL}$ of $n-\mathrm{C}_{6} \mathrm{~F}_{13} \mathrm{I}$ was heated for $48 \mathrm{~h}$ at $100{ }^{\circ} \mathrm{C}$ under argon. Workup of the reaction mixture as above and column chromatography $\left(\mathrm{SiO}_{2} /\right.$ pentane) gave $62 \mathrm{mg}(0.19 \mathrm{mmol}, 38 \%)$ of 1-bromo-3-fluoro-5-iodocubane (3) as a colorless liquid. ${ }^{1} \mathrm{H}$ NMR: $4.78(\mathrm{~m}, 1 \mathrm{H}), 4.66(\mathrm{~m}, 1 \mathrm{H}), 4.61(\mathrm{~m}, 1 \mathrm{H}), 4.30(\mathrm{~m}, 1 \mathrm{H}), 4.17$ (m, 1H). ${ }^{13} \mathrm{C}$ NMR: 98.7 (d, $J=330 \mathrm{~Hz}, \mathrm{C}), 73.2(\mathrm{~d}, J=24 \mathrm{~Hz}, \mathrm{CH}), 66.0$ (d, $J=14 \mathrm{~Hz}, \mathrm{CH}), 65.8$ (d, $J=24 \mathrm{~Hz}, \mathrm{CH}), 65.6$ (d, $J=24 \mathrm{~Hz}, \mathrm{CH}), 51.6(\mathrm{~d}, J=7 \mathrm{~Hz}, \mathrm{C}), 37.3$ (d, $J=5 \mathrm{~Hz}, \mathrm{CH}), 18.6$ (d, $J=7 \mathrm{~Hz}, \mathrm{C}) . \mathrm{MS}(M / z)$ : 246, 200, 198, 181, 179, 176, 174, 152, 127, 120 (100\%), 101, 100, 99, 95, 94, 75, 74, 70, 64, $63,62,51$.

1-Chloro-3-fluoro-5-iodocubane (4). A solution of $80 \mathrm{mg}(0.51 \mathrm{mmol})$ of 1-chloro-3fluoro cubane $(\mathbf{8}), 15 \mathrm{mg}(0.06 \mathrm{mmol})$ of iron(II) acetyl acetonate, $10 \mathrm{mg}(0.04 \mathrm{mmol})$ of dibenzoyl peroxide, and $0.3 \mathrm{~mL}$ of acetic acid in $4 \mathrm{~mL}$ of $n-\mathrm{C}_{6} \mathrm{~F}_{13} \mathrm{I}$, was heated for $48 \mathrm{~h}$ at $100{ }^{\circ} \mathrm{C}$ under argon. Workup of the reaction mixture as above and column chromatography $\left(\mathrm{SiO}_{2} /\right.$ pentane) gave $48 \mathrm{mg}$ (33\%) of 1-chloro-3-fluoro-5-iodocubane (4) as a colorless liquid. ${ }^{1} \mathrm{H}$ NMR: $4.73(\mathrm{~m}, 1 \mathrm{H}), 4.59(\mathrm{~m}, 2 \mathrm{H}), 4.24(\mathrm{~m}, 1 \mathrm{H}), 4.05(\mathrm{~m}, 1 \mathrm{H}) .{ }^{13} \mathrm{C} \mathrm{NMR}: 97.6$ (d, $J=330$ Hz, C), 73.1 (d, J=24 Hz, CH), 66.0 (d, J=24 Hz, CH), 65.8 (d, J=14 Hz, CH), $65.2(\mathrm{~d}, J=24$ $\mathrm{Hz}, \mathrm{CH}), 63.1$ (d, $J=7 \mathrm{~Hz}, \mathrm{C}), 35.5$ (d, $J=5 \mathrm{~Hz}, \mathrm{CH}), 17.5$ (d, $J=8 \mathrm{~Hz}, \mathrm{C}) . \mathrm{MS}(M / z): 283,282$, 
247, 238, 222, 212, 154, 152, 137, 135, 121, 120 (100\%), 119, 101, 100. 99, 94, 95, 75, 74, 60, $59,58,51,50$.

1-Bromo-3-chloro-5-fluoro-7-iodocubane (5). A solution of $75 \mathrm{mg}(0.23 \mathrm{mmol})$ of 1bromo-3-fluoro-5-iodo cubane (3), $950 \mathrm{mg}(9.1 \mathrm{mmol})$ and of $t$-BuOCl in $5 \mathrm{~mL}$ of $\mathrm{CCl}_{4}$ was irradiated for $12 \mathrm{~h}$ at $0{ }^{\circ} \mathrm{C}$ with $150 \mathrm{~W}$ high-pressure $\mathrm{Hg}$-lamp. Workup of the reaction mixture as above and column chromatography $\left(\mathrm{SiO}_{2} /\right.$ pentane $)$ gave $17 \mathrm{mg}(0.047 \mathrm{mmol}, 20 \%)$ of 1-bromo3-chloro-5-fluoro-7-iodocubane (5) as a white solid, m.p. $=138-139{ }^{\circ} \mathrm{C} .{ }^{1} \mathrm{H}$ NMR: $4.79(\mathrm{~m}, 1 \mathrm{H})$, 4.69 (m, 2H), 4.38 (m, 1H). ${ }^{13} \mathrm{C}$ NMR: 94.3 (d, J = 331 Hz, C), 73.4 (d, J = 24 Hz, CH), 73.2 (d, $J=24 \mathrm{~Hz}, \mathrm{CH}), 73.1(\mathrm{~d}, J=24 \mathrm{~Hz}, \mathrm{CH}), 72.5(\mathrm{~d}, J=14 \mathrm{~Hz}, \mathrm{CH}), 59.4(\mathrm{~d}, J=8 \mathrm{~Hz}, \mathrm{C}), 45.5$ (d, $J$ $=8 \mathrm{~Hz}, \mathrm{C}), 10.3$ (d, $J=8 \mathrm{~Hz}, \mathrm{C})$. MS (M/z): 360, 326, 324, 282, 280, 246, 200, 156, 154 (100\%), $127,119,118,110,99,98,93,74,69,63,57$.

1-Chloro-3-fluorocubane (8). A solution of $200 \mathrm{mg}(1.64 \mathrm{mmol})$ of fluorocubane (7), $240 \mathrm{mg}$ of $t$ - BuOCl $(2.2 \mathrm{mmol}), 6 \mathrm{~mL}$ of $\mathrm{CCl}_{4}$ irradiated for $20 \mathrm{~min}$ at $0{ }^{\circ} \mathrm{C}$ with $150 \mathrm{~W}$ highpressure Hg-lamp. Workup of the reaction mixture as above and column chromatography $\left(\mathrm{SiO}_{2} /\right.$ pentane) gave $94 \mathrm{mg}(0.60 \mathrm{mmol}, 37 \%)$ of 1-chloro-3-fluoro cubane $(\mathbf{8})$ as a white solid (m. p. $\left.=38-39{ }^{\circ} \mathrm{C}\right) .{ }^{1} \mathrm{H}$ NMR: $4.48(\mathrm{~m}, 2 \mathrm{H}), 4.37(\mathrm{~m}, 1 \mathrm{H}), 4.07(\mathrm{~m}, 1 \mathrm{H}), 3.83(\mathrm{~m}, 2 \mathrm{H}) .{ }^{13} \mathrm{C}$ NMR: $99.2(\mathrm{~d}, J=330 \mathrm{~Hz}, \mathrm{C}), 65.4(\mathrm{~d}, J=5 \mathrm{~Hz}, \mathrm{C}), 65.0(\mathrm{~d}, J=24 \mathrm{~Hz}, \mathrm{CH}), 57.5(\mathrm{~d}, J=14 \mathrm{~Hz}, \mathrm{CH})$, $57.0(\mathrm{~d}, J=24 \mathrm{~Hz}, \mathrm{CH}), 35.8(\mathrm{~J}=5 \mathrm{~Hz}, \mathrm{CH}) . \mathrm{MS}(M / z): 155,154,136,132,130,121,120,102$, $101(100 \%), 96,95,94,74,73,70,63,57,51,50,44,39,37$.

1-Bromo-3-fluorocubane (9). A solution of $250 \mathrm{mg}$ ( $2 \mathrm{mmol}$ ) of fluorocubane (7), 650 $\mathrm{mg}(4.2 \mathrm{mmol})$ of $t-\mathrm{BuOBr}$, and $1 \mathrm{~g}(3 \mathrm{mmol})$ of $\mathrm{CBr}_{4}$ in $10 \mathrm{~mL}$ of $\mathrm{CF}_{2} \mathrm{Br}_{2}$ was irradiated for 30 min at $0{ }^{\circ} \mathrm{C}$ with $150 \mathrm{~W}$ high-pressure Hg-lamp. Workup of the reaction mixture as above and column chromatography $\left(\mathrm{SiO}_{2} /\right.$ pentane $)$ gave $175 \mathrm{mg}(0.87 \mathrm{mmol}, 42 \%)$ of 1-fluoro-3bromocubane (9) as a white solid, m. p. $=35-36{ }^{\circ} \mathrm{C} .{ }^{1} \mathrm{H}$ NMR: $4.59(\mathrm{~m}, 2 \mathrm{H}), 4.39(\mathrm{~m}, 1 \mathrm{H}), 4.12$ ( m, 1H), 3.93 (m, 2H). ${ }^{13} \mathrm{C}$ NMR: $100.4(\mathrm{~d}, J=330 \mathrm{~Hz}, \mathrm{C}), 65.6$ (d, $\left.J=24 \mathrm{~Hz}, \mathrm{CH}\right), 58.1$ (d, $J=$ $14 \mathrm{~Hz}, \mathrm{CH}), 56.9$ (d, $J=24 \mathrm{~Hz}, \mathrm{CH}), 55.3(\mathrm{~d}, J=8 \mathrm{~Hz}, \mathrm{C}), 37.6(\mathrm{~d}, J=5 \mathrm{~Hz}, \mathrm{CH}) . \mathrm{MS}(\mathrm{M} / z)$ : 202, 200, 199, 176, 174, 121, 120, 102, 101 (100\%), 87, 86, 81, 77, 75, 74, 70, 69, 63, 60, 57, $51,50,39$. 


\subsection{X-ray structural determinations}

1-Bromo-3-chloro-5-iodocubane (2). X-ray data for 2 were collected at $100 \mathrm{~K}$ on a Bruker SMART ${ }^{\mathrm{TM}}$ Apex II X-ray diffractometer system with graphite-monochromated Mo-K $\mathrm{K}_{\alpha}$ radiation, using the omega/phi-scan technique. The data were collected in a hemisphere of data in 1664 frames with 10 second exposure times. Cell parameters and an orientation matrix for data collection corresponded to a orthorhombic crystal system: $a=11.7218(6) \AA, b=10.5234(5)$ $\AA, c=7.3143(4) \AA, V=902.24(8) \AA^{3}, D_{\text {calcd }}=2.528 \mathrm{~g} \mathrm{~cm}^{-3}$, and $Z=4$. The asymmetric unit contains one molecule of the form $\mathrm{C}_{8} \mathrm{H}_{4} \mathrm{IBrCl}$. The structure was solved in the space group Pnma (No. 62) by direct methods using the SHELXTL 6.1 bundled software package. The heavy atom positions were determined using direct methods employing the SHELXTL routine methods. The remaining non-hydrogen atoms were located from successive difference Fourier map calculations. In the final cycles of each refinement, the non-hydrogen atoms were refined anisotropically. The two atoms of $\mathrm{Cl}, \mathrm{Br}$ are found disordered at three positions of atoms $\mathrm{C}(1)$, $\mathrm{C}(5)$, and $\mathrm{C}(5 \mathrm{~A})$ while the atom I disordered at two positions of atoms $\mathrm{C}(5), \mathrm{C}(5)$. The occupancies of $\mathrm{Cl}, \mathrm{Br}$ and I atoms are as follows: $\mathrm{C}(1): \mathrm{Cl}(2)$ 0.35978, $\mathrm{Br}(2)$ 0.64024; $\mathrm{C}(5)$ : $\mathrm{Cl}(1)$ 0.32011, Br(1) 0.17988, I(1) 0.49999; C(5A): Cl(1A) 0.32011, Br(1A) 0.17988, I(1A) 0.49999. Hydrogen atom positions were calculated and allowed to ride on the carbon to which they are bonded assuming a C-H bond length of $0.95 \AA$. Using 1042 observed reflections $(I>2 \sigma(I))$, refinement converged at $\mathrm{R} 1=0.0369$ and $\mathrm{wR} 2=0.0870$.

1-Bromo-3-chloro-5-fluoro-7-iodocubane (5). X-ray data for 5 were collected at $100 \mathrm{~K}$ on a Bruker SMART ${ }^{\mathrm{TM}}$ Apex II X-ray diffractometer system with graphite-monochromated Mo$\mathrm{K}_{\alpha}$ radiation, using the omega/phi-scan technique. The data were collected in a hemisphere of data in 1664 frames with 10 second exposure times. Cell parameters and an orientation matrix for data collection corresponded to a triclinic crystal system: $a=6.2544(2) \AA, b=8.2784(3) \AA, c$ $=9.9866(5) \AA, \alpha=87.453(2)^{\circ}, \beta=97.760(3)^{\circ}, \gamma=68.020(2)^{\circ}, V=473.38(3) \AA^{3}, D_{\text {calcd }}=2.535 \mathrm{~g}$ $\mathrm{cm}^{-3}$, and $Z=2$. The asymmetric unit contains one molecule of the form $\mathrm{C}_{8} \mathrm{H}_{4} \mathrm{IBrClF}$. The structure was solved in the space group $P-1$ (No. 2) by direct methods using the SHELXTL 6.1 bundled software package. The three atoms of $\mathrm{Cl}, \mathrm{Br}$ and $\mathrm{I}$ are found disordered at three carbon atoms: $\mathrm{C}(3), \mathrm{C}(6)$, and $\mathrm{C}(8)$. The occupancies of $\mathrm{Cl}, \mathrm{Br}$ and $\mathrm{I}$ atoms for compound 5 is as follows: C(3): $\mathrm{Cl}(3)$ 0.55370, $\mathrm{Br}(3)$ 0.42952, I(3) 0.01678; C(6): $\mathrm{Cl}(1)$ 0.18708, $\mathrm{Br}(1)$ 0.35841, I(1) 0.45451; $\mathrm{C}(8): \mathrm{Cl}(2)$ 0.25922, $\mathrm{Br}(2)$ 0.21207, I(2) 0.52870. Hydrogen atom positions were 
calculated and allowed to ride on the carbon to which they are bonded assuming a $\mathrm{C}-\mathrm{H}$ bond length of $0.95 \AA$ A. Using 1620 observed reflections $(I>2 \sigma(I))$, refinement converged at R1 = 0.0323 and $\mathrm{wR} 2=0.0743$.

\section{Computational details}

Full Gaussian reference: Frisch, M. J.; Trucks, G. W.; Schlegel, H. B.; Scuseria, G. E.; Robb, M. A.; Cheeseman, J. R.; Montgomery, J., J. A.; Vreven, T.; Kudin, K. N.; Burant, J. C.; Millam, J. M.; Iyengar, S. S.; Tomasi, J.; Barone, V.; Mennucci, B.; Cossi, M.; Scalmani, G.; Rega, N.; Petersson, G. A.; Nakatsuji, H.; Hada, M.; Ehara, M.; Toyota, K.; Fukuda, R.; Hasegawa, J.; Ishida, M.; Nakajima, T.; Honda, Y.; Kitao, O.; Nakai, H.; Klene, M.; Li, X.; Knox, J. E.; Hratchian, H. P.; Cross, J. B.; Adamo, C.; Jaramillo, J.; Gomperts, R.; Stratmann, R. E.; Yazyev, O.; Austin, A. J.; Cammi, R.; Pomelli, C.; Ochterski, J. W.; Ayala, P. Y.; Morokuma, K.; Voth, G. A.; Salvador, P.; Dannenberg, J. J.; Zakrzewski, V. G.; Dapprich, S.; Daniels, A. D.; Strain, M. C.; Farkas, O.; Malick, D. K.; Rabuck, A. D.; Raghavachari, K.; Foresman, J. B.; Ortiz, J. V.; Cui, Q.; Baboul, A. G.; Clifford, S.; Cioslowski, J.; Stefanov, B. B.; Liu, G.; Liashenko, A.; Piskorz, P.; Komaromi, I.; Martin, R. L.; Fox, D. J.; Keith, T.; Al-Laham, M. A.; Peng, C. Y.; Nanayakkara, A.; Challacombe, M.; Gill, P. M. W.; Johnson, B.; Chen, W.; Wong, M. W.; Gonzalez, C.; Pople, J. A.; B.03 ed.: Pittsburgh, 2003.

\subsection{Computations of parity violation potentials}

Parity violating potentials in compounds 1-5 were computed within the two-component quasirelativistic Hartree-Fock approach to electroweak quantum chemistry outlined in Ref. 13 of the communication. This approach employs the zeroth order regular approximation (ZORA). In order to alleviate the gauge dependence of ZORA, van Wüllens model potential approach ${ }^{1}$ was utilized. The atomic model densities $\rho_{\mathrm{A}}{ }^{\bmod }(r)$ employed in this study for generating the model potentials are given by $\rho_{\mathrm{A}}{ }^{\bmod }(r)=\pi^{-3 / 2} \Sigma_{i} \mathrm{c}_{i A}{ }^{\bmod }\left[\alpha_{i A}{ }^{\bmod }\right]^{3 / 2} \exp \left[-\alpha_{i A}{ }^{\bmod } r^{2}\right]$ and the coefficients and exponents of the various atomic model densities are listed in Table S14-S19. The basis set used herein correspond to those given in Ref. 13: For elements of the second row of the periodic table (here $\mathrm{C}$ and $\mathrm{F}$ ) the large uncontracted even-tempered basis set given in Ref. 13 for $\mathrm{O}$ was employed, for $\mathrm{Cl}$ the basis set for $\mathrm{S}$, for $\mathrm{Br}$ the basis set for $\mathrm{Se}$ and for I the basis set for Te. On hydrogen the augmented correlation consistent polarized valence double zeta set (aug-cc-pVDZ) ${ }^{2}$ 
was used in uncontracted form. Dense grids (in total 32000 radial shells) were used for the numerical integration involved in the computation of the matrix elements of the parity violating operator and the ZORA kinetic energy operator. Fermi's constant $G_{\mathrm{F}}$ was employed with the value $2.22254 \cdot 10^{-14} E_{\mathrm{h}} a_{0}$ and the Weinberg parameter with $\sin ^{2}\left(\theta_{\mathrm{w}}\right)=0.2319$. A Gaussian nuclear model with exponents $\alpha_{Z}^{\text {nuc }}(A)$ was used with $\alpha_{Z}^{\text {nuc }}(A)=3 /\left[2\left(r^{\text {nuc }}(A)\right)^{2}\right]$ and $r^{\text {nuc }}(A)=(0.836$ $\left.A^{1 / 3}+0.570\right) \mathrm{fm} . \quad A$ is the atomic mass number of the specific isotope with atomic number $Z$. Parity violating potentials have been computed for the isotopomers containing the isotopes ${ }^{1} \mathrm{H}$, ${ }^{12} \mathrm{C},{ }^{19} \mathrm{~F},{ }^{35} \mathrm{Cl},{ }^{79} \mathrm{Br}$ and ${ }^{127} \mathrm{I}$. A negative value of a parity violating potential $V_{\mathrm{pv}}$ indicates that the given structure is stabilized with respect to its mirror image due to the parity violating weak interaction. The energy difference between the two structures is two times the parity violating potential.

Parity violating vibrational frequency shifts were computed as described $\mathrm{in}^{3}$ in the separable anharmonic adiabatic approximation. Briefly, one-dimensional cuts through the ("ordinary") parity conserving $\left(V_{\mathrm{pc}}(\boldsymbol{q})\right)$ and the parity violating $\left(V_{\mathrm{pv}}(\boldsymbol{q})\right)$ potential energy hypersurfaces were computed along selected dimensionless reduced normal coordinates $q_{i}$ of the $(S)$-enantiomer of $\mathbf{1}$. The parity conserving potential computed with a step size of 0.05 in the interval $-3 \leq q_{i} \leq 3$ was used to solve numerically the one-dimensional vibrational Schrödinger equation on a grid. ${ }^{4}$ The resulting ground $\left|0_{i}\right\rangle$ and first excited $\left|1_{i}\right\rangle$ vibrational states, respectively, were used to compute for each state the expectation value $\left\langle v_{i}\left|V_{\mathrm{pv}}\left(q_{i}\right) /(h c)\right| v_{i}\right\rangle$ of the coordinate dependent parity violating potential $V_{\mathrm{pv}}\left(q_{i}\right)$, which beforehand was fitted to a third order polynomial in $q_{i}$ according to

$$
V_{\mathrm{pv}}\left(q_{i}\right) /(h c)=\sum_{j=1}^{3} p_{j} q_{i}^{j} .
$$

The relative parity violating vibrational frequency difference is then obtained via $\Delta_{\mathrm{pv}} n_{i} / n_{i}=$ $2\left(\left\langle 1_{i}\left|V_{\mathrm{pv}}\left(q_{i}\right) /(h c)\right| 1_{i}\right\rangle-\left\langle 0_{i}\left|V_{\mathrm{pv}}\left(q_{i}\right) /(h c)\right| 0_{i}\right\rangle\right) / x_{i}$, with $x_{i}$ being the wavenumber term difference between the first excited and the ground vibrational level in mode $i$. In the harmonic approximation this term difference corresponds to $\tilde{\omega}_{i}$, while anharmonically the vibrational fundamental is denoted as $\tilde{v}_{i}$. 
Table S10 lists the polynomial fit coefficients $p_{i}$ of the parity violating potential $V_{\mathrm{pv}}$ along two modes with significant C-F stretching contributions. Tables S12-S14 reports the individual contributions to the relative parity violating vibrational frequency splittings.

For comparison with the polyhalomethanes the parity violating vibrational frequency shifts of modes with considerable $\mathrm{C}-\mathrm{F}$ contributions $\left(\widetilde{\omega}=946,1164\right.$, and $\left.1351 \mathrm{~cm}^{-1}\right)$ were computed (Tables S12-S14) in the separable anharmonic adiabatic approximation. ${ }^{3}$ The usually quite intense $\mathrm{C}-\mathrm{F}$ stretching mode of the polyhalomethanes has received particular attention because the corresponding fundamental typically falls in the wave number range at which $\mathrm{CO}_{2}$ lasers operate (900 to $1100 \mathrm{~cm}^{-1}$ ). The absolute value of the relative shift in $\mathbf{1}$, however, is about one to two orders of magnitude smaller than the relative shifts in the $\mathrm{C}-\mathrm{F}$ stretching mode of $\mathrm{CHBrClF}$. Thus, the lowering of the parity violating vibrational frequency shift of the C-F stretching mode by at least one order of magnitude when going from the methane to the cubane derivative parallels the reduction in the parity violating energy difference. Such a link between the size of relative parity violating energy differences and of relative rovibrational frequency shifts has already been proposed by Letokhov. ${ }^{5}$ This relation may also be used to estimate the expected parity violating vibrational frequency shifts in the remaining chiral polyhalocubanes.

\section{Additional References}

(1) van Wüllen, C. J. Chem. Phys. 1998, 109, 392-399.

(2) Dunning Jr., T. H.; Peterson, K. A.; Woon, D. E. In The Encyclopedia of Computational Chemistry; Schleyer, P. v. R., Allinger, N. L., Clark, T., Gasteiger, J., Kollman, P. A., Schaefer, H. F., Schreiner, P. R., Eds.; John Wiley \& Sons: Chichester, 1998; Vol. 1, pp 88-115; Dunning Jr., T. H. J. Chem. Phys. 1989, 90, 1007-1023.

(3) Quack, M.; Stohner, J. Z. Phys. Chem. 2000, 214, 675-703.

(4) Meyer, R. J. Chem. Phys. 1970, 52, 2053-2059.

(5) Letokhov, V. S. Phys. Lett. A 1975, 53, 275-276. 
Table S1. Unscaled harmonic wavenumbers $(\widetilde{\omega})$ and integrated infrared band strengths $(G)$ of 1 calculated on the B3LYP/6-311G* level of theory. Integrated band strength were calculated in the double harmonic approximation.

$\begin{array}{lll}\text { Mode } & \widetilde{\omega} / \mathrm{cm}^{-1} & G / \mathrm{pm}^{2} \\ v_{1} & 3166 & 0.0101 \\ v_{2} & 3163 & 0.0691 \\ v_{3} & 3160 & 0.1063 \\ v_{4} & 3158 & 0.0784 \\ v_{5} & 3142 & 0.0927 \\ v_{6} & 1352 & 2.7345 \\ v_{7} & 1244 & 1.0273 \\ v_{8} & 1233 & 0.4078 \\ v_{9} & 1204 & 0.1780 \\ v_{10} & 1203 & 0.4423 \\ v_{11} & 1164 & 0.2059 \\ v_{12} & 1135 & 0.1442 \\ v_{13} & 1107 & 0.0249 \\ v_{14} & 1105 & 0.0118 \\ v_{15} & 1074 & 0.0003 \\ v_{16} & 1070 & 0.0080 \\ v_{17} & 1063 & 0.0350 \\ v_{18} & 1042 & 0.0156 \\ v_{19} & 1041 & 0.0541 \\ v_{20} & 1030 & 0.0062 \\ v_{21} & 988 & 0.0233 \\ v_{22} & 973 & 0.0214 \\ v_{23} & 966 & 0.0327 \\ v_{24} & 947 & 1.0443 \\ v_{25} & 881 & 0.4409 \\ v_{26} & 855 & 0.1778 \\ v_{27} & 851 & 0.0533 \\ v_{28} & 849 & 0.1164 \\ v_{29} & 815 & 0.1115 \\ v_{30} & 779 & 1.0262 \\ v_{31} & 706 & 0.0129 \\ v_{32} & 677 & 0.0057 \\ v_{33} & 676 & 0.0099 \\ v_{34} & 522 & 0.4008 \\ v_{35} & 383 & 0.7544 \\ v_{36} & 333 & 0.0516 \\ v_{37} & 308 & 0.2432 \\ v_{38} & 276 & 0.3295 \\ v_{39} & 239 & 0.0154 \\ v_{40} & 169 & 0.1622 \\ v_{41} & 143 & 0.1181 \\ v_{42} & 105 & 0.0682\end{array}$


Table S2. Reference geometry of the $(S)$-enantiomer of $\mathbf{1}$ (in $\AA$ ) in the center of mass system.

$\begin{array}{lrrr}\mathrm{C} & -0.5898291744 & -0.9804989306 & -0.7954818256 \\ \mathrm{C} & 0.0213169402 & 0.6611163413 & 2.1744582601 \\ \mathrm{C} & -1.2041714606 & 1.1216241369 & -0.3686837812 \\ \mathrm{C} & -0.4343783044 & 0.0024755491 & 0.4132515830 \\ \mathrm{C} & 0.7695275380 & -0.4490320568 & -1.3605621134 \\ \mathrm{C} & 0.1655787098 & 1.6127419321 & -0.9414336458 \\ \mathrm{C} & -1.3429297169 & 0.1435831073 & -1.5909584564 \\ \mathrm{C} & 0.9385362792 & 0.5163123537 & -0.1391744668 \\ \mathrm{Cl} & 2.0639198116 & -1.4719465557 & -2.0070255089 \\ \mathrm{Br} & -0.7531743891 & -0.4654439404 & 2.2626250795 \\ \mathrm{~F} & 0.5501375525 & 2.9133446125 & -1.0116859577 \\ \mathrm{H} & -1.9863084574 & 1.7539763622 & 0.0423650488 \\ \mathrm{H} & 0.2214411557 & 0.9241163191 & -3.2096472401 \\ \mathrm{H} & -0.8765628471 & -2.0258741216 & -0.7272539560 \\ \mathrm{H} & 1.8474143963 & 0.6582716017 & 0.4389369372 \\ \mathrm{H} & -2.2513272230 & -0.0051187100 & -2.1692019822\end{array}$

Table S3. Cartesian displacements (in $\AA$ ) corresponding to a unit shift along the dimensionless reduced normal coordinate $q_{6}$ of the $(S)$-enantiomer of compound $\mathbf{1 .}$

$\begin{array}{lrrr}\mathrm{C} & -0.000841959961 & -0.002123237976 & -0.000007335898 \\ \mathrm{C} & 0.002378471087 & 0.008053887939 & 0.002799125429 \\ \mathrm{C} & 0.005342823062 & 0.006949770137 & -0.001416521851 \\ \mathrm{C} & 0.000653369271 & 0.002263874191 & -0.000565400911 \\ \mathrm{C} & 0.000275840221 & 0.002546741155 & 0.000005789146 \\ \mathrm{C} & -0.010372982230 & -0.034886721071 & 0.001849199577 \\ \mathrm{C} & 0.000130611788 & 0.002767410314 & -0.000617240277 \\ \mathrm{C} & -0.000121054097 & 0.008107331643 & -0.002017318385 \\ \mathrm{Cl} & 0.000094309880 & -0.000185485175 & -0.000056471991 \\ \mathrm{Br} & -0.000016988428 & -0.000045685770 & 0.000058174176 \\ \mathrm{~F} & 0.003078143348 & 0.010267196462 & -0.000528162060 \\ \mathrm{H} & -0.019181322298 & -0.029492930572 & 0.004876157449 \\ \mathrm{H} & -0.009725282603 & -0.032925419595 & -0.008757976821 \\ \mathrm{H} & -0.000023086371 & -0.002634024173 & 0.000733125386 \\ \mathrm{H} & -0.000919765456 & -0.032422114215 & 0.007273590580 \\ \mathrm{H} & 0.000302216870 & -0.010795659415 & 0.002874756285\end{array}$

Table S4. Cartesian displacements (in $\AA$ ) corresponding to a unit shift along the dimensionless reduced normal coordinate $q_{11}$ of the $(S)$-enantiomer of compound $\mathbf{1}$

$\begin{array}{lrrr}\mathrm{C} & -0.006347791185 & -0.003789862377 & -0.000874952550 \\ \mathrm{C} & -0.003861973218 & -0.010336454779 & -0.003632129252 \\ \mathrm{C} & -0.007758684521 & -0.010085841452 & 0.003091950622 \\ \mathrm{C} & 0.006430451773 & 0.008671483088 & -0.018565452859 \\ \mathrm{C} & -0.010787937071 & 0.015233338559 & 0.007661198188 \\ \mathrm{C} & -0.001100488101 & -0.011629815627 & 0.001218646585 \\ \mathrm{C} & 0.014668884914 & 0.008360029859 & 0.007340864455 \\ \mathrm{C} & -0.002453383619 & -0.009591935742 & 0.002660116371 \\ \mathrm{Cl} & 0.001099712205 & -0.000949556783 & -0.000610027867 \\ \mathrm{Br} & -0.000104166938 & -0.000131860988 & 0.000475917369 \\ \mathrm{~F} & 0.001680871286 & 0.006306381707 & -0.000421296570 \\ \mathrm{H} & 0.023709502292 & 0.037386082189 & -0.011552187397 \\ \mathrm{H} & 0.012741112825 & 0.036393428199 & 0.012144328598 \\ \mathrm{H} & 0.018208485288 & -0.009797573193 & 0.005003064934 \\ \mathrm{H} & 0.001228339564 & 0.033086642846 & -0.015509889495 \\ \mathrm{H} & 0.015912908736 & -0.015875240941 & 0.014850491636\end{array}$


Table S5. Cartesian displacements (in $\AA$ ) corresponding to a unit shift along the dimensionless reduced normal coordinate $q_{24}$ of the $(S)$-enantiomer of compound $\mathbf{1}$.

$\begin{array}{lrrr}\text { C } & 0.007983182924 & 0.004153208370 & 0.004745729152 \\ \mathrm{C} & -0.002152974354 & -0.008255022543 & -0.002351403779 \\ \mathrm{C} & -0.006440454203 & -0.005140916058 & -0.001597592854 \\ \mathrm{C} & -0.004353740328 & -0.011818157688 & 0.014264983182 \\ \mathrm{C} & 0.011514764912 & -0.017229101316 & -0.010839679131 \\ \mathrm{C} & 0.002898278585 & -0.013789217088 & 0.006062765615 \\ \mathrm{C} & -0.009301478382 & 0.010663187690 & -0.006894028893 \\ \mathrm{C} & 0.000584046513 & -0.004706055966 & 0.001513842923 \\ \mathrm{Cl} & -0.002136880015 & 0.002293795455 & 0.001241664961 \\ \mathrm{Br} & 0.000160881517 & 0.000377909928 & -0.000702748800 \\ \mathrm{~F} & 0.003075084081 & 0.014256674776 & -0.001456019474 \\ \mathrm{H} & 0.010328295809 & 0.032489482275 & -0.026721970702 \\ \mathrm{H} & -0.011340882578 & 0.043921422577 & 0.008816649591 \\ \mathrm{H} & 0.009449889161 & 0.004263226514 & 0.017509452376 \\ \mathrm{H} & -0.004299958167 & 0.078201298546 & -0.010195532651 \\ \mathrm{H} & -0.009270630538 & 0.012360066599 & -0.008413070548\end{array}$

Table S6a. One dimensional cuts through the parity conserving potential energy hypersurface $\left(V_{\mathrm{pc}}\right.$ in Hartree $\left.\left(E_{\mathrm{h}}\right)\right)$ along selected dimensionless reduced normal coordinates of $(S)-\mathbf{1}$ with C-F stretching contributions. $V_{\mathrm{pv}}$ was computed on the B3LYP/6-311G* level of theory.

$\begin{array}{llll}q_{i} & V_{\mathrm{pc}}\left(q_{6}\right) / E_{\mathrm{h}} & V_{\mathrm{pc}}\left(q_{11}\right) / E_{\mathrm{h}} & V_{\mathrm{pc}}\left(q_{24}\right) / E_{\mathrm{h}} \\ -4.00 & -3441.90786855 & -3441.92324288 & -3441.92936393 \\ -3.95 & -3441.90948106 & -3441.92432955 & -3441.93033352 \\ -3.90 & -3441.91106602 & -3441.92540202 & -3441.93128889 \\ -3.85 & -3441.91262370 & -3441.92646032 & -3441.93223010 \\ -3.80 & -3441.91415437 & -3441.92750445 & -3441.93315720 \\ -3.75 & -3441.91565827 & -3441.92853443 & -3441.93407025 \\ -3.70 & -3441.91713566 & -3441.92955027 & -3441.93496932 \\ -3.65 & -3441.91858680 & -3441.93055199 & -3441.93585446 \\ -3.60 & -3441.92001193 & -3441.93153960 & -3441.93672574 \\ -3.55 & -3441.92141129 & -3441.93251312 & -3441.93758319 \\ -3.50 & -3441.92278513 & -3441.93347256 & -3441.93842690 \\ -3.45 & -3441.92413367 & -3441.93441794 & -3441.93925691 \\ -3.40 & -3441.92545716 & -3441.93534926 & -3441.94007328 \\ -3.35 & -3441.92675582 & -3441.93626656 & -3441.94087608 \\ -3.30 & -3441.92802987 & -3441.93716983 & -3441.94166535 \\ -3.25 & -3441.92927954 & -3441.93805910 & -3441.94244116 \\ -3.20 & -3441.93050505 & -3441.93893438 & -3441.94320357 \\ -3.15 & -3441.93170661 & -3441.93979568 & -3441.94395263 \\ -3.10 & -3441.93288443 & -3441.94064303 & -3441.94468840 \\ -3.05 & -3441.93403872 & -3441.94147642 & -3441.94541094 \\ -3.00 & -3441.93516969 & -3441.94229589 & -3441.94612030 \\ -2.95 & -3441.93627753 & -3441.94310144 & -3441.94681655 \\ -2.90 & -3441.93736246 & -3441.94389309 & -3441.94749973 \\ -2.85 & -3441.93842465 & -3441.94467086 & -3441.94816992 \\ -2.80 & -3441.93946431 & -3441.94543475 & -3441.94882715 \\ -2.75 & -3441.94048163 & -3441.94618478 & -3441.94947150 \\ -2.70 & -3441.94147679 & -3441.94692097 & -3441.95010301 \\ -2.65 & -3441.94244997 & -3441.94764333 & -3441.95072174 \\ -2.60 & -3441.94340136 & -3441.94835188 & -3441.95132775 \\ -2.55 & -3441.94433114 & -3441.94904663 & -3441.95192109 \\ -2.50 & -3441.94523947 & -3441.94972760 & -3441.95250181 \\ -2.45 & -3441.94612653 & -3441.95039479 & -3441.95306998 \\ -2.40 & -3441.94699250 & -3441.95104823 & -3441.95362564\end{array}$




\begin{tabular}{|c|c|c|c|}
\hline-2.35 & -3441.94783753 & -3441.95168792 & -3441.95416884 \\
\hline-2.30 & -3441.94866179 & -3441.95231389 & -3441.95469964 \\
\hline-2.25 & -3441.94946544 & -3441.95292614 & -3441.95521810 \\
\hline-2.20 & -3441.95024864 & -3441.95352469 & -3441.95572426 \\
\hline-2.15 & -3441.95101155 & -3441.95410956 & -3441.95621818 \\
\hline-2.10 & -3441.95175432 & -3441.95468075 & -3441.95669990 \\
\hline-2.05 & -3441.95247711 & -3441.95523828 & -3441.95716947 \\
\hline-2.00 & -3441.95318005 & -3441.95578217 & -3441.95762695 \\
\hline-1.95 & -3441.95386331 & -3441.95631242 & -3441.95807238 \\
\hline-1.90 & -3441.95452701 & -3441.95682905 & -3441.95850582 \\
\hline-1.85 & -3441.95517131 & -3441.95733208 & -3441.95892730 \\
\hline-1.80 & -3441.95579634 & -3441.95782151 & -3441.95933688 \\
\hline-1.75 & -3441.95640224 & -3441.95829736 & -3441.95973460 \\
\hline-1.70 & -3441.95698915 & -3441.95875964 & -3441.96012051 \\
\hline-1.65 & -3441.95755719 & -3441.95920836 & -3441.96049465 \\
\hline-1.60 & -3441.95810651 & -3441.95964355 & -3441.96085706 \\
\hline-1.55 & -3441.95863722 & -3441.96006519 & -3441.96120779 \\
\hline-1.50 & -3441.95914946 & -3441.96047332 & -3441.96154688 \\
\hline-1.45 & -3441.95964336 & -3441.96086794 & -3441.96187437 \\
\hline-1.40 & -3441.96011902 & -3441.96124907 & -3441.96219031 \\
\hline-1.35 & -3441.96057658 & -3441.96161670 & -3441.96249472 \\
\hline-1.30 & -3441.96101616 & -3441.96197087 & -3441.96278766 \\
\hline-1.25 & -3441.96143787 & -3441.96231157 & -3441.96306915 \\
\hline-1.20 & -3441.96184182 & -3441.96263882 & -3441.96333924 \\
\hline-1.15 & -3441.96222814 & -3441.96295262 & -3441.96359797 \\
\hline-1.10 & -3441.96259692 & -3441.96325300 & -3441.96384536 \\
\hline-1.05 & -3441.96294829 & -3441.96353995 & -3441.96408145 \\
\hline-1.00 & -3441.96328235 & -3441.96381349 & -3441.96430628 \\
\hline-0.95 & -3441.96359921 & -3441.96407363 & -3441.96451988 \\
\hline-0.90 & -3441.96389897 & -3441.96432038 & -3441.96472228 \\
\hline-0.85 & -3441.96418174 & -3441.96455374 & -3441.96491352 \\
\hline-0.80 & -3441.96444761 & -3441.96477373 & -3441.96509361 \\
\hline-0.75 & -3441.96469670 & -3441.96498035 & -3441.96526260 \\
\hline-0.70 & -3441.96492908 & -3441.96517362 & -3441.96542050 \\
\hline-0.65 & -3441.96514487 & -3441.96535354 & -3441.96556735 \\
\hline-0.60 & -3441.96534416 & -3441.96552012 & -3441.96570317 \\
\hline-0.55 & -3441.96552704 & -3441.96567337 & -3441.96582800 \\
\hline-0.50 & -3441.96569360 & -3441.96581329 & -3441.96594184 \\
\hline-0.45 & -3441.96584394 & -3441.96593989 & -3441.96604473 \\
\hline-0.40 & -3441.96597814 & -3441.96605318 & -3441.96613668 \\
\hline-0.35 & -3441.96609629 & -3441.96615317 & -3441.96621773 \\
\hline-0.30 & -3441.96619848 & -3441.96623986 & -3441.96628788 \\
\hline-0.25 & -3441.96628479 & -3441.96631326 & -3441.96634716 \\
\hline-0.20 & -3441.96635531 & -3441.96637337 & -3441.96639558 \\
\hline-0.15 & -3441.96641011 & -3441.96642020 & -3441.96643317 \\
\hline-0.10 & -3441.96644928 & -3441.96645375 & -3441.96645993 \\
\hline-0.05 & -3441.96647291 & -3441.96647404 & -3441.96647589 \\
\hline+0.00 & -3441.96648105 & -3441.96648105 & -3441.96648105 \\
\hline+0.05 & -3441.96647381 & -3441.96647481 & -3441.96647544 \\
\hline+0.10 & -3441.96645124 & -3441.96645531 & -3441.96645905 \\
\hline+0.15 & -3441.96641342 & -3441.96642256 & -3441.96643190 \\
\hline+0.20 & -3441.96636043 & -3441.96637655 & -3441.96639401 \\
\hline+0.25 & -3441.96629234 & -3441.96631730 & -3441.96634537 \\
\hline+0.30 & -3441.96620922 & -3441.96624480 & -3441.96628600 \\
\hline+0.35 & -3441.96611114 & -3441.96615907 & -3441.96621590 \\
\hline+0.40 & -3441.96599816 & -3441.96606009 & -3441.96613508 \\
\hline
\end{tabular}




\begin{tabular}{|c|c|c|c|}
\hline+0.45 & -3441.96587036 & -3441.96594787 & -3441.96604354 \\
\hline+0.50 & -3441.96572780 & -3441.96582242 & -3441.96594129 \\
\hline+0.55 & -3441.96557055 & -3441.96568373 & -3441.96582833 \\
\hline+0.60 & -3441.96539867 & -3441.96553181 & -3441.96570465 \\
\hline+0.65 & -3441.96521222 & -3441.96536666 & -3441.96557027 \\
\hline+0.70 & -3441.96501127 & -3441.96518827 & -3441.96542517 \\
\hline+0.75 & -3441.96479587 & -3441.96499665 & -3441.96526936 \\
\hline+0.80 & -3441.96456610 & -3441.96479180 & -3441.96510284 \\
\hline+0.85 & -3441.96432199 & -3441.96457371 & -3441.96492560 \\
\hline+0.90 & -3441.96406363 & -3441.96434238 & -3441.96473764 \\
\hline+0.95 & -3441.96379105 & -3441.96409782 & -3441.96453895 \\
\hline+1.00 & -3441.96350433 & -3441.96384003 & -3441.96432952 \\
\hline+1.05 & -3441.96320350 & -3441.96356899 & -3441.96410936 \\
\hline+1.10 & -3441.96288864 & -3441.96328471 & -3441.96387844 \\
\hline+1.15 & -3441.96255979 & -3441.96298719 & -3441.96363677 \\
\hline+1.20 & -3441.96221701 & -3441.96267642 & -3441.96338434 \\
\hline+1.25 & -3441.96186035 & -3441.96235240 & -3441.96312112 \\
\hline+1.30 & -3441.96148986 & -3441.96201512 & -3441.96284712 \\
\hline+1.35 & -3441.96110559 & -3441.96166459 & -3441.96256232 \\
\hline+1.40 & -3441.96070759 & -3441.96130080 & -3441.96226671 \\
\hline+1.45 & -3441.96029592 & -3441.96092375 & -3441.96196027 \\
\hline+1.50 & -3441.95987061 & -3441.96053342 & -3441.96164298 \\
\hline+1.55 & -3441.95943173 & -3441.96012982 & -3441.96131485 \\
\hline+1.60 & -3441.95897932 & -3441.95971294 & -3441.96097584 \\
\hline+1.65 & -3441.95851341 & -3441.95928278 & -3441.96062595 \\
\hline+1.70 & -3441.95803408 & -3441.95883933 & -3441.96026515 \\
\hline+1.75 & -3441.95754134 & -3441.95838258 & -3441.95989344 \\
\hline+1.80 & -3441.95703526 & -3441.95791253 & -3441.95951078 \\
\hline+1.85 & -3441.95651588 & -3441.95742917 & -3441.95911716 \\
\hline+1.90 & -3441.95598324 & -3441.95693250 & -3441.95871257 \\
\hline+1.95 & -3441.95543739 & -3441.95642250 & -3441.95829698 \\
\hline+2.00 & -3441.95487836 & -3441.95589918 & -3441.95787037 \\
\hline+2.05 & -3441.95430621 & -3441.95536252 & -3441.95743273 \\
\hline+2.10 & -3441.95372097 & -3441.95481251 & -3441.95698402 \\
\hline+2.15 & -3441.95312268 & -3441.95424916 & -3441.95652423 \\
\hline+2.20 & -3441.95251139 & -3441.95367244 & -3441.95605334 \\
\hline+2.25 & -3441.95188714 & -3441.95308236 & -3441.95557132 \\
\hline+2.30 & -3441.95124997 & -3441.95247891 & -3441.95507816 \\
\hline+2.35 & -3441.95059992 & -3441.95186207 & -3441.95457382 \\
\hline+2.40 & -3441.94993703 & -3441.95123183 & -3441.95405829 \\
\hline+2.45 & -3441.94926133 & -3441.95058820 & -3441.95353154 \\
\hline+2.50 & -3441.94857287 & -3441.94993115 & -3441.95299355 \\
\hline+2.55 & -3441.94787168 & -3441.94926069 & -3441.95244429 \\
\hline+2.60 & -3441.94715780 & -3441.94857679 & -3441.95188374 \\
\hline+2.65 & -3441.94643128 & -3441.94787945 & -3441.95131188 \\
\hline+2.70 & -3441.94569214 & -3441.94716867 & -3441.95072868 \\
\hline+2.75 & -3441.94494043 & -3441.94644442 & -3441.95013412 \\
\hline+2.80 & -3441.94417617 & -3441.94570671 & -3441.94952817 \\
\hline+2.85 & -3441.94339942 & -3441.94495552 & -3441.94891081 \\
\hline+2.90 & -3441.94261019 & -3441.94419083 & -3441.94828202 \\
\hline+2.95 & -3441.94180853 & -3441.94341265 & -3441.94764176 \\
\hline+3.00 & -3441.94099448 & -3441.94262095 & -3441.94699002 \\
\hline+3.05 & -3441.94016806 & -3441.94181573 & -3441.94632677 \\
\hline+3.10 & -3441.93932932 & -3441.94099698 & -3441.94565198 \\
\hline+3.15 & -3441.93847829 & -3441.94016468 & -3441.94496564 \\
\hline+3.20 & -3441.93761499 & -3441.93931883 & -3441.94426772 \\
\hline
\end{tabular}




$\begin{array}{llll}+3.25 & -3441.93673947 & -3441.93845941 & -3441.94355819 \\ +3.30 & -3441.93585175 & -3441.93758641 & -3441.94283703 \\ +3.35 & -3441.93495188 & -3441.93669982 & -3441.94210421 \\ +3.40 & -3441.93403988 & -3441.93579963 & -3441.94135972 \\ +3.45 & -3441.93311579 & -3441.93488582 & -3441.94060353 \\ +3.50 & -3441.93217964 & -3441.93395839 & -3441.93983561 \\ +3.55 & -3441.93123145 & -3441.93301733 & -3441.93905595 \\ +3.60 & -3441.93027127 & -3441.93206261 & -3441.93826452 \\ +3.65 & -3441.92929912 & -3441.93109424 & -3441.93746129 \\ +3.70 & -3441.92831504 & -3441.93011219 & -3441.93664625 \\ +3.75 & -3441.92731906 & -3441.92911645 & -3441.93581937 \\ +3.80 & -3441.92631120 & -3441.92810702 & -3441.93498064 \\ +3.85 & -3441.92529150 & -3441.92708388 & -3441.93413002 \\ +3.90 & -3441.92425998 & -3441.92604702 & -3441.93326751 \\ +3.95 & -3441.92321669 & -3441.92499642 & -3441.93239307 \\ +4.00 & -3441.92216164 & -3441.92393208 & -3441.93150669\end{array}$

Table S6b. One dimensional cut through the parity violating potential energy hypersurface $\left(V_{\mathrm{pv}}\right.$ in $\left.10^{-20} E_{\mathrm{h}}\right)$ along the dimensionless reduced normal coordinate $q_{6}$ of the $(S)$-enantiomer of compound $\mathbf{1}$. $V_{\mathrm{pv}}$ was computed within the quasirelativistic two-component zeroth order regular approximation approach.

$\begin{array}{ll}q_{6} & V_{\mathrm{pv}}\left(q_{6}\right) /\left(10^{-20} E_{\mathrm{h}}\right) \\ -3.00 & -28.094 \\ -2.50 & -25.231 \\ -2.00 & -21.834 \\ -1.50 & -18.677 \\ -1.00 & -15.730 \\ -0.75 & -14.326 \\ -0.50 & -12.966 \\ -0.25 & -11.647 \\ +0.00 & -10.364 \\ +0.25 & -9.116 \\ +0.50 & -7.900 \\ +0.75 & -6.711 \\ +1.00 & -5.551 \\ +1.50 & -3.301 \\ +2.00 & -1.126 \\ +2.50 & 0.991 \\ +3.00 & 3.073\end{array}$

Table S7. One dimensional cut through the parity violating potential energy hypersurface $\left(V_{\mathrm{pv}}\right.$ in $\left.10^{-20} E_{\mathrm{h}}\right)$ along the dimensionless reduced normal coordinate $q_{11}$ of the $(S)$-enantiomer of compound $\mathbf{1}$. $V_{\mathrm{pv}}$ was computed within the quasirelativistic two-component zeroth order regular approximation approach.

$\begin{array}{ll}q_{11} & V_{\mathrm{pv}}\left(q_{11}\right) /\left(10^{-20} E_{\mathrm{h}}\right) \\ -2.00 & -14.218 \\ -1.50 & -12.831 \\ -1.00 & -11.728 \\ -0.75 & -11.283 \\ -0.50 & -10.908 \\ -0.25 & -10.601 \\ +0.00 & -10.364 \\ +0.25 & -10.195 \\ +0.50 & -10.090 \\ +0.75 & -10.052 \\ +1.00 & -10.077 \\ +1.50 & -10.317 \\ +2.00 & -10.801\end{array}$


Table S8. One dimensional cut through the parity violating potential energy hypersurface $\left(V_{\mathrm{pv}}\right.$ in $\left.10^{-20} E_{\mathrm{h}}\right)$ along the dimensionless reduced normal coordinate $q_{24}$ of the $(S)$-enantiomer of compound $\mathbf{1}$. $V_{\mathrm{pv}}$ was computed within the quasirelativistic two-component zeroth order regular approximation approach.

$\begin{array}{lr}q_{24} & V_{\mathrm{pv}}\left(q_{24}\right) /\left(10^{-20} E_{\mathrm{h}}\right) \\ -3.00 & -107.118 \\ -2.50 & -91.350 \\ -2.00 & -75.333 \\ -1.50 & -59.145 \\ -1.00 & -42.866 \\ -0.75 & -34.718 \\ -0.50 & -26.578 \\ -0.25 & -18.457 \\ -0.15 & -15.216 \\ +0.00 & -10.364 \\ +0.15 & -5.526 \\ +0.25 & -2.309 \\ +0.50 & 5.698 \\ +0.75 & 13.649 \\ +1.00 & 21.536 \\ +1.50 & 37.081 \\ +2.00 & 52.275 \\ +2.50 & 67.069 \\ +3.00 & 81.423\end{array}$

Table S9a. Polynomial fit coefficients $p_{j}$ and root mean square deviation $d_{\text {RMS }}$ (all in $10^{-15} \mathrm{~cm}^{-1}$ ) of the parity violating potential $V_{\mathrm{pv}}$ of the $(S)$-enantiomer of compound $\mathbf{1}$. The standard deviations due to the fit procedure are given in parenthesis in units of the last significant digits.

$\begin{array}{llllll}\text { Mode } & p_{0} & p_{1} & p_{2} & p_{3} & d_{\text {RMS }} \\ v_{6} & -22.734(6) & 11.099(6) & -0.6210(14) & 0.0662(9) & 0.003 \\ v_{11} & -22.7495(11) & 1.7903(17) & -1.1771(6) & 0.0212(5) & 0.003 \\ v_{24} & -22.782(16) & 70.872(19) & -0.610(4) & -0.2123(29) & 0.049\end{array}$

Table S9b. Individual contributions to the parity violating vibrational wavenumber shift in the mode $v_{6}$ of the $(S)$ enantiomer of compound $\mathbf{1}$ computed in the separable anharmonic adiabatic approximation. All values are given in $10^{-15} \mathrm{~cm}^{-1}$. With a calculated anharmonic vibrational wavenumber of $\tilde{v}_{6}=1354.3 \mathrm{~cm}^{-1}$ one obtains a relative parity violating vibrational wavenumber splitting of $4.0 \cdot 10^{-20}$.

$\begin{array}{llll}j & \left\langle 1\left|p_{j} q^{j}\right| 1\right\rangle & \left\langle 0\left|p_{j} q^{j}\right| 0\right\rangle & \left\langle 1\left|p_{j} q^{j}\right| 1\right\rangle-\left\langle 0\left|p_{j} q^{j}\right| 0\right\rangle \\ 0 & -22.7342 & -22.7342 & 0.0000 \\ 1 & 0.8414 & 0.291 & 0.5500 \\ 2 & -0.9314 & -0.3102 & -0.6212 \\ 3 & 0.0198 & 0.0032 & 0.0166 \\ \text { Sum } & -22.8044 & -22.7498 & 0.0546\end{array}$

Table S10. Individual contributions to the parity violating vibrational wavenumber shift in the mode $v_{11}$ of the $(S)$ enantiomer of compound $\mathbf{1}$ computed in the separable anharmonic adiabatic approximation. All values are given in $10^{-15} \mathrm{~cm}^{-1}$. With a calculated anharmonic vibrational wavenumber of $\tilde{v}_{11}=1165.5 \mathrm{~cm}^{-1}$ one obtains a relative parity violating vibrational wavenumber splitting of $2.01 \cdot 10^{-18}$.

$\begin{array}{lccc}j & \left\langle 1\left|p_{j} q^{j}\right| 1\right\rangle & \left\langle 0\left|p_{j} q^{j}\right| 0\right\rangle & \left\langle 1\left|p_{j} q^{j}\right| 1\right\rangle-\left\langle 0\left|p_{j} q^{j}\right| 0\right\rangle \\ 0 & -22.7495 & -22.7495 & 0.0000 \\ 1 & 0.0107 & 0.0054 & 0.0053 \\ 2 & -1.7624 & -0.5879 & -1.1745 \\ 3 & 0.0005 & 0.0001 & 0.0004 \\ \text { Sum } & -24.5007 & -23.3319 & -1.1688\end{array}$


Table S11. Individual contributions to the parity violating vibrational wavenumber shift in the mode $v_{24}$ of the $(S)$ enantiomer of compound $\mathbf{1}$ computed in the separable anharmonic adiabatic approximation. All values are given in $10^{-15} \mathrm{~cm}^{-1}$. With a calculated anharmonic vibrational wavenumber of $950.8 \mathrm{~cm}^{-1}$ one obtains a relative parity violating vibrational wavenumber splitting of $3.45 \cdot 10^{-19}$.

$\begin{array}{lccc}j & \left\langle 1\left|p_{j} q^{j}\right| 1\right\rangle & \left\langle 0\left|p_{j} q^{j}\right| 0\right\rangle & \left\langle 1\left|p_{j} q^{j}\right| 1\right\rangle-\left\langle 0\left|p_{j} q^{j}\right| 0\right\rangle \\ 0 & -22.783 & -22.783 & 0.000 \\ 1 & 1.100 & 0.320 & 0.780 \\ 2 & -0.909 & -0.304 & -0.605 \\ 3 & -0.013 & -0.002 & -0.011 \\ \text { Total } & -22.605 & -22.769 & 0.164\end{array}$

Table S12. Parameters of the model density for hydrogen used to construct the model potential in the twocomponent ZORA approach.
$\alpha_{i}^{\text {mod }}$
$\mathrm{C}_{i}^{\text {mod }}$
17.388049797
0.0059257005
3. 615672064
0.0701929046
1.131622661
0.2942023101
0.414384954
0.4675289539
0.164265903
0.1621501309

Table S12: Parameters of the model density for carbon used to construct the model potential in the two-component ZORA approach.

\begin{tabular}{rr}
\multicolumn{1}{l}{$\alpha_{i}{ }^{\bmod }$} & \multicolumn{1}{l}{${ }^{\bmod }$} \\
2070.774305545 & 0.0010923571 \\
461.404488918 & 0.0131848517 \\
152.608754693 & 0.0825787292 \\
59.791280065 & 0.3185641540 \\
25.876222409 & 0.7140266003 \\
11.859459978 & 0.7316712413 \\
5.087813417 & 0.0606645033 \\
2.969226350 & -0.2268722274 \\
1.211297436 & 1.0008540944 \\
0.598767703 & 1.9692870936 \\
0.280515660 & 1.1619425008 \\
0.119495557 & 0.1730061016
\end{tabular}

Table S13: Parameters of the model density for fluorine used to construct the model potential in the two-component ZORA approach.

\begin{tabular}{rr}
\multicolumn{1}{c}{$\alpha_{i}^{\text {mod }}$} & \multicolumn{1}{l}{${ }^{\bmod }$} \\
4419.253086854 & 0.0013029633 \\
960.909783424 & 0.0162869683 \\
314.157764313 & 0.1013887891 \\
123.001778863 & 0.3765722564 \\
53.566098852 & 0.7705042247 \\
24.780284877 & 0.6341543993 \\
11.568626848 & -0.1642026549 \\
5.544792664 & 0.1508323748 \\
2.695204902 & 2.1674479967 \\
1.278526970 & 3.0381335655 \\
0.581993664 & 1.6487215029 \\
0.249186416 & 0.2588576140
\end{tabular}


Table S14: Parameters of the model density for chlorine used to construct the model potential in the two-component ZORA approach.

$$
\begin{array}{rr}
\multicolumn{1}{l}{\alpha_{i}^{\bmod }} & \multicolumn{1}{l}{{ }^{\bmod }} \\
19287.804681839 & 0.0010713781 \\
4020.200134141 & 0.0137467966 \\
1303.675237194 & 0.0855561608 \\
515.518210978 & 0.3237621562 \\
228.924324710 & 0.7073948633 \\
110.227592116 & 0.6592431646 \\
45.560289938 & -0.1879459248 \\
19.446917686 & 0.6508221368 \\
12.060796874 & 3.4101257134 \\
6.680633765 & 3.9496006910 \\
2.000865011 & -1.8777781585 \\
1.248998860 & 2.2026653269 \\
0.627555462 & 4.3155618935 \\
0.315937949 & 2.4154191526 \\
0.152254250 & 0.3307546495
\end{array}
$$

Table S15: Parameters of the model density for bromine used to construct the model potential in the two-component ZORA approach.

\begin{tabular}{rr}
\multicolumn{1}{l}{$\alpha_{i}^{\bmod }$} & \multicolumn{1}{l}{${ }^{\bmod }$} \\
182464.36304025 & \multicolumn{1}{c}{0.0004367396} \\
40843.980479792 & 0.0032730734 \\
16297.184129947 & 0.0135304741 \\
6717.723584933 & 0.0673806117 \\
2808.709419944 & 0.2545751550 \\
1261.531647450 & 0.6177770422 \\
604.565906839 & 0.7404132330 \\
276.374189024 & -0.0498473994 \\
146.581616615 & -0.3930437919 \\
89.243376413 & 2.7124752668 \\
49.964040905 & 5.4261428194 \\
17.539290386 & -3.7611828044 \\
9.874183490 & 4.3778614712 \\
5.842686566 & 10.8836811610 \\
3.382342236 & 5.4262886413 \\
1.653923883 & -1.0507343871 \\
1.074325744 & 1.7315214134 \\
0.553362063 & 4.6803521179 \\
0.279120303 & 2.9138897944 \\
0.135308392 & 0.4052093686
\end{tabular}


Table S16: Parameters of the model density for iodine used to construct the model potential in the two-component ZORA approach.

\begin{tabular}{rr}
\multicolumn{1}{l}{$\alpha_{i}{ }^{\bmod }$} & \multicolumn{1}{l}{${ }_{1}{ }^{\bmod }$} \\
1152328.4026025 & 0.0002152463 \\
206153.01514185 & 0.0018612039 \\
74394.431161075 & 0.0059463239 \\
35063.961002930 & 0.0214513402 \\
14998.727194114 & 0.0902900967 \\
6572.126604388 & 0.2833471666 \\
3082.118621454 & 0.6049251012 \\
1534.279537615 & 0.6815519257 \\
683.890382230 & -0.0206572962 \\
441.876370298 & -0.3600658248 \\
239.923702849 & 2.3747495110 \\
141.699905325 & 5.6982349309 \\
48.582203508 & -10.8354852030 \\
39.418201864 & 8.1871902725 \\
20.877154399 & 20.1678059591 \\
13.564718336 & 2.9912604509 \\
8.362039353 & -11.8718401976 \\
3.942023464 & 11.8556340146 \\
2.444980732 & 12.0605433627 \\
1.163326507 & 1.5512300749 \\
0.714651751 & -2.5012549377 \\
0.586886446 & 5.5839864204 \\
0.333011606 & 3.7995937450 \\
0.200561695 & 2.3010127693 \\
0.106851364 & 0.3284735436
\end{tabular}


xyz-coordinates of all computed structures (B3LYP/6-311G*/MIDI! on iodine) in $\AA$ :

$$
\begin{aligned}
& 16 \\
& \text { Bromochlorofluorocubane (1) } \\
& \text { C-0.594858412-0.997882950-0.695459576 } \\
& \text { C } 0.016287703 \quad 0.643732322-2.074436010 \\
& \text { C-1.209200698 1.104240118-0.268661531 } \\
& \text { C- } 0.439407542-0.014908470 \quad 0.513273833 \\
& \text { C } 0.764498301-0.466416076-1.260539864 \\
& \text { C } 0.1605494731 .595357913-0.841411396 \\
& \text { C-1.347958954 0.126199088-1.490936207 } \\
& \text { C } 0.9335070420 .498928334-0.039152217 \\
& \text { Cl 2.058890574-1.489330575-1.907003259 } \\
& \mathrm{Br}-0.758203626-0.4828279602 .362647329 \\
& \text { F } 0.5451083152 .895960593-0.911663708 \\
& \mathrm{H}-1.991337695 \quad 1.736592343 \quad 0.142387299 \\
& \text { H } 0.2164119190 .906732300-3.109624990 \\
& \mathrm{H}-0.881592084-2.043258141-0.627231706 \\
& \text { H } 1.842385159 \quad 0.640887582 \quad 0.538959187 \\
& \mathrm{H}-2.256356460-0.022502729-2.069179732 \\
& 16 \\
& \text { Bromochloroiodocubane (2) } \\
& \text { C-0.889858465-0.272897181 } 0.112216337 \\
& \text { C-0.240013037 1.801283407-0.407572162 } \\
& \begin{array}{llll}
\text { C } 1.116052749 & 0.475547448 & 0.759240288
\end{array} \\
& \text { C-0.132235908-0.139788333 } 1.474997541 \\
& \text { C-1.466770938 } 1.165430901 \quad 0.326659723 \\
& \begin{array}{lllll}
\text { C } 0.511942143 & 1.909313888 & 0.965843838
\end{array} \\
& \text { C } 0.3510692590 .362699272-0.604231170 \\
& \text { C-0.729004791 } 1.289619730 \quad 1.701448143 \\
& \mathrm{Cl}-3.156647481 \quad 1.614854014 \quad 0.044738729 \\
& \mathrm{Br}-0.057121296-1.5261100322 .821244733 \\
& \text { H } 2.147037727 \quad 0.181498491 \quad 0.931678890 \\
& \mathrm{H}-0.2987459392 .571354237-1.171106534 \\
& \mathrm{H}-1.452794311-1.138742016-0.223157511 \\
& \text { I } 1.212207741-0.370291930-2.432887983 \\
& \mathrm{H}-1.177021990 \quad 1.646916956 \quad 2.624025617 \\
& \text { H } 1.069680709 \quad 2.778522713 \quad 1.305883376 \\
& 16 \\
& \text { Bromofluoroiodocubane (3) } \\
& \text { C-0.624945037-1.072598117-0.468275288 } \\
& \text { C } 1.605354042-1.073814016-0.469823009 \\
& \text { C } 0.4921748380 .867918875-0.467285007 \\
& \text { C-0.605314331 } 0.207555693-1.368296857 \\
& \text { C } 0.487815536-1.707995277-1.373667453 \\
& \text { C } 1.583485502 \quad 0.205384481-1.368612230 \\
& \text { C } 0.490135265-0.4240548790 .422108603 \\
& \text { C 0.490350099-0.430609729-2.288829915 } \\
& \mathrm{Br}-2.1815458571 .127023160-2.011224500 \\
& \text { F 2.690375309 0.850189987-1.819450670 } \\
& \mathrm{H} 0.4890793701 .893295153-0.108576698 \\
& \text { H 2.495887999-1.576395710-0.102646955 } \\
& \mathrm{H}-1.518086833-1.569745748-0.101759997 \\
& \text { I } 0.494082469-0.4174543192 .575233365 \\
& \text { H } 0.486835332-0.416825616-3.375297536 \\
& \text { H } 0.486305018-2.733491858-1.735086681
\end{aligned}
$$


16

Chlorofluoroiodocubane (4)

C-1.257831450-0.359775941 0.749195578

C $0.979691669-0.361318177 \quad 0.748178419$

C-0.144673592 $1.574057020 \quad 0.749639461$

C-1.252409207 $0.922577846 \quad 1.653564527$

C-0.139795588-0.981889113 1.649701449

C $0.951802058 \quad 0.915643429 \quad 1.648453513$

C-0.139681965 0.282824346-0.142127029

C-0.144952352 $0.286393697 \quad 2.569230199$

Cl-0.131170016-2.653117574 2.239083422

F 2.064460429 1.551114748 2.098582206

$\mathrm{H}-0.134130028 \quad 2.596700933 \quad 0.382850372$

H $1.865773979-0.8786118450 .390971494$

$\mathrm{H}-2.134497125-0.8870692860 .384667413$

I-0.127994851 $0.281742196-2.293118478$

$\mathrm{H}-0.135058543 \quad 0.274723949 \quad 3.655786433$

H-2.141512188 $1.433807226 \quad 2.014328894$

16

Bromochlorofluoroiodocubane (5)

C-0.673961170-0.739737204-0.273408080

C $1.562645721-0.738637945-0.275117195$

C $0.4450654041 .198652936-0.273292323$

C-0.653243761 $0.539185482-1.175748552$

C 0.442686135-1.360787821-1.176641699

C $1.537870804 \quad 0.538992178-1.175874567$

C $0.443849405-0.094123784 \quad 0.616646776$

C $0.444586020-0.093458950-2.097766410$

Cl 0.444436196-3.026205606-1.768482185

$\mathrm{Br}-2.2274115581 .449107808-1.822381831$

F 2.643795258 1.176370578-1.629722440

H $0.440365365 \quad 2.222793450 \quad 0.086908332$

H 2.447835350-1.255960947 0.082108423

$\mathrm{H}-1.560192763-1.2540447420 .084276213$

I $0.444234646-0.093889177 \quad 2.762876991$

$\mathrm{H} 0.440396359-0.097783885-3.183498001$ 


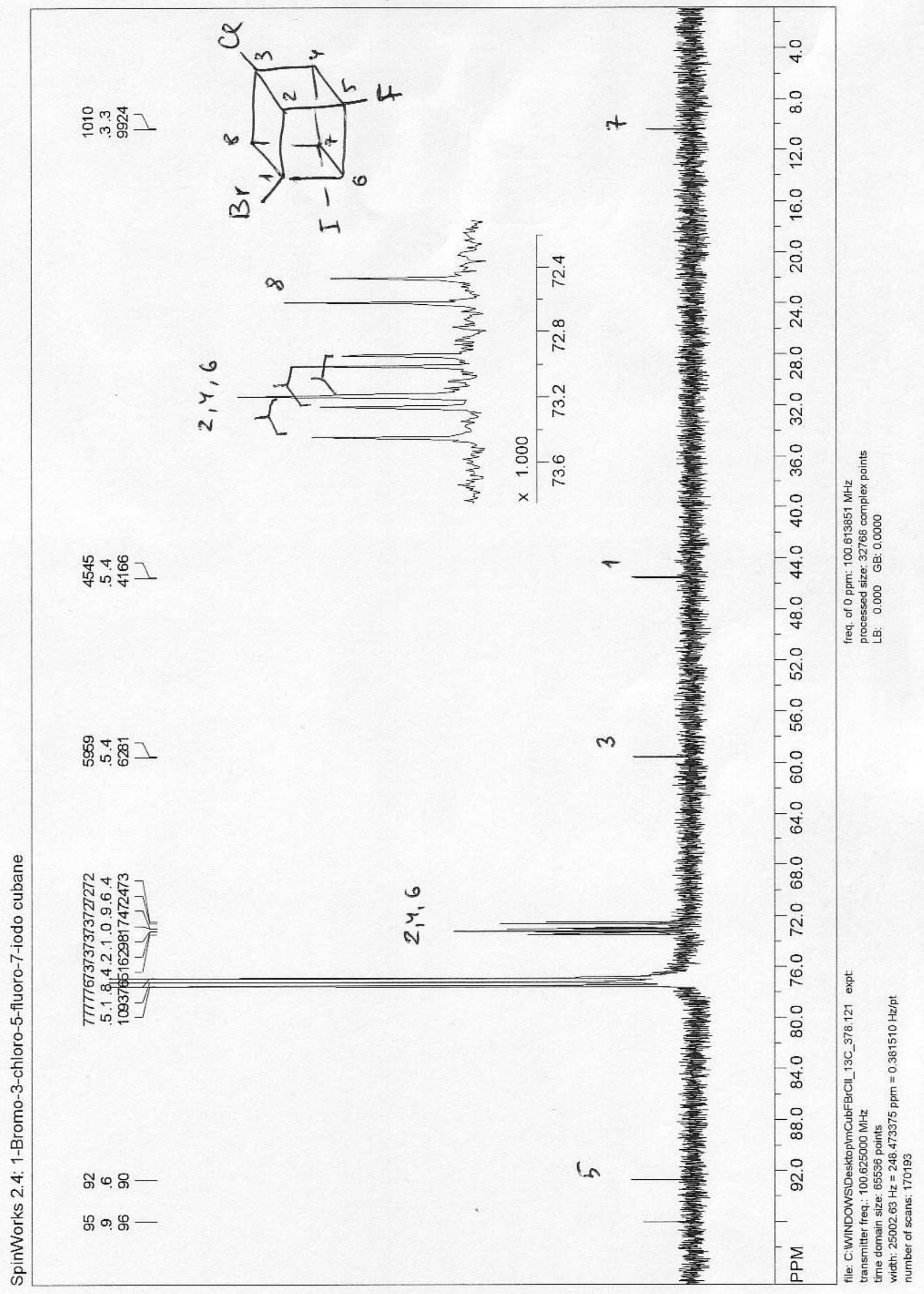




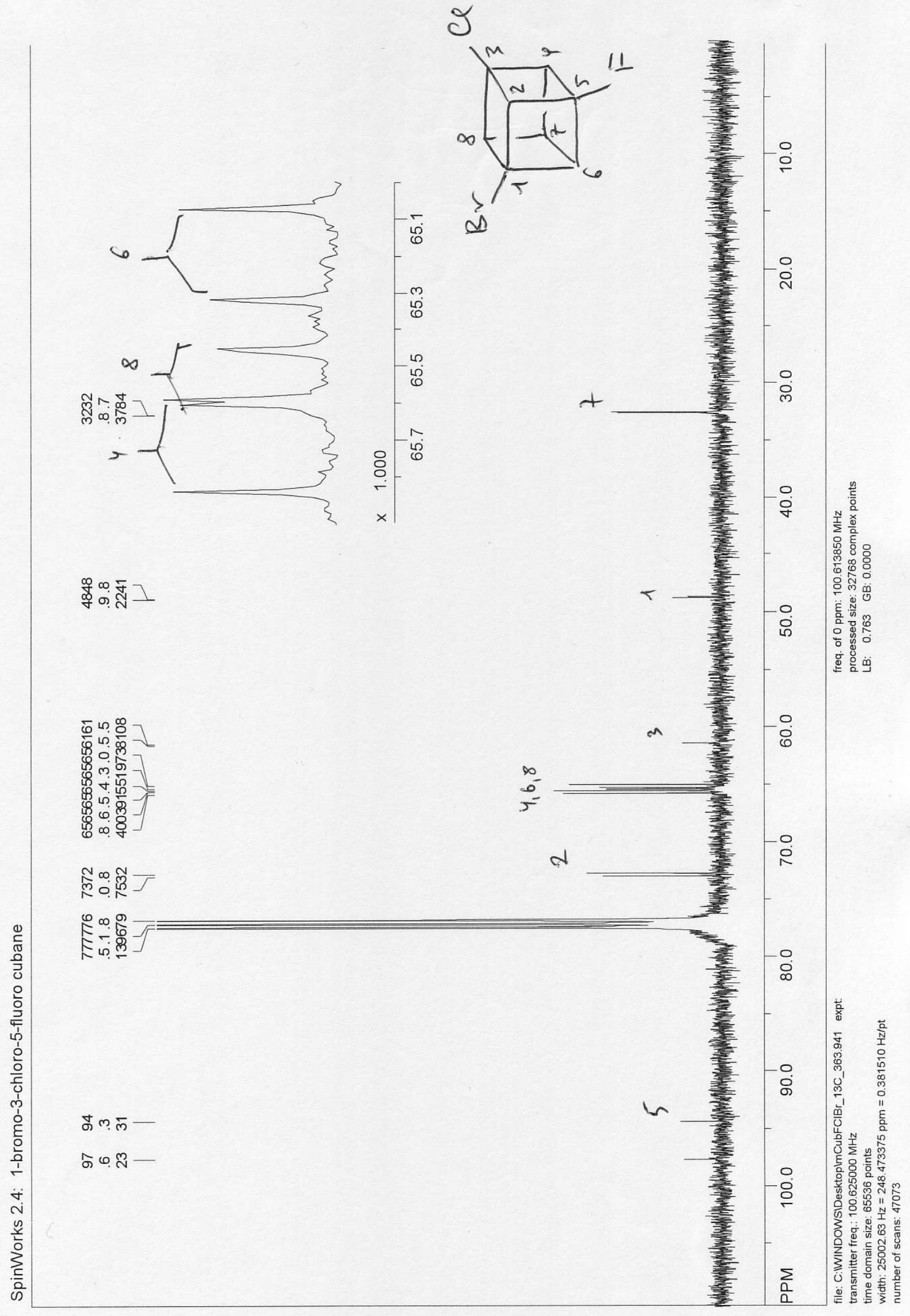




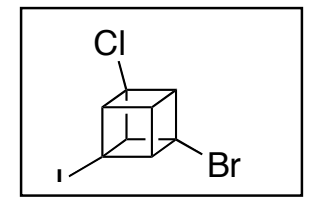

-

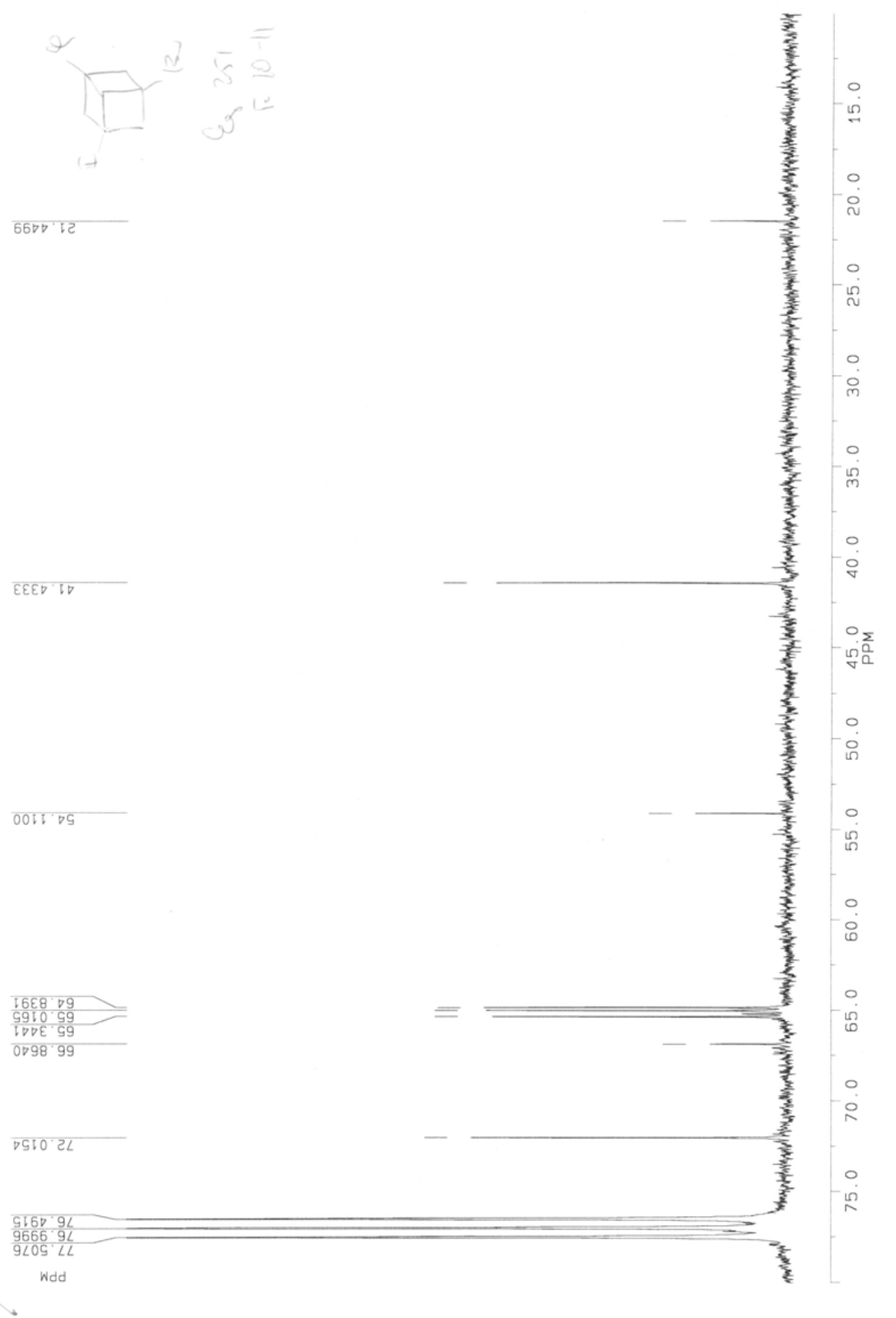




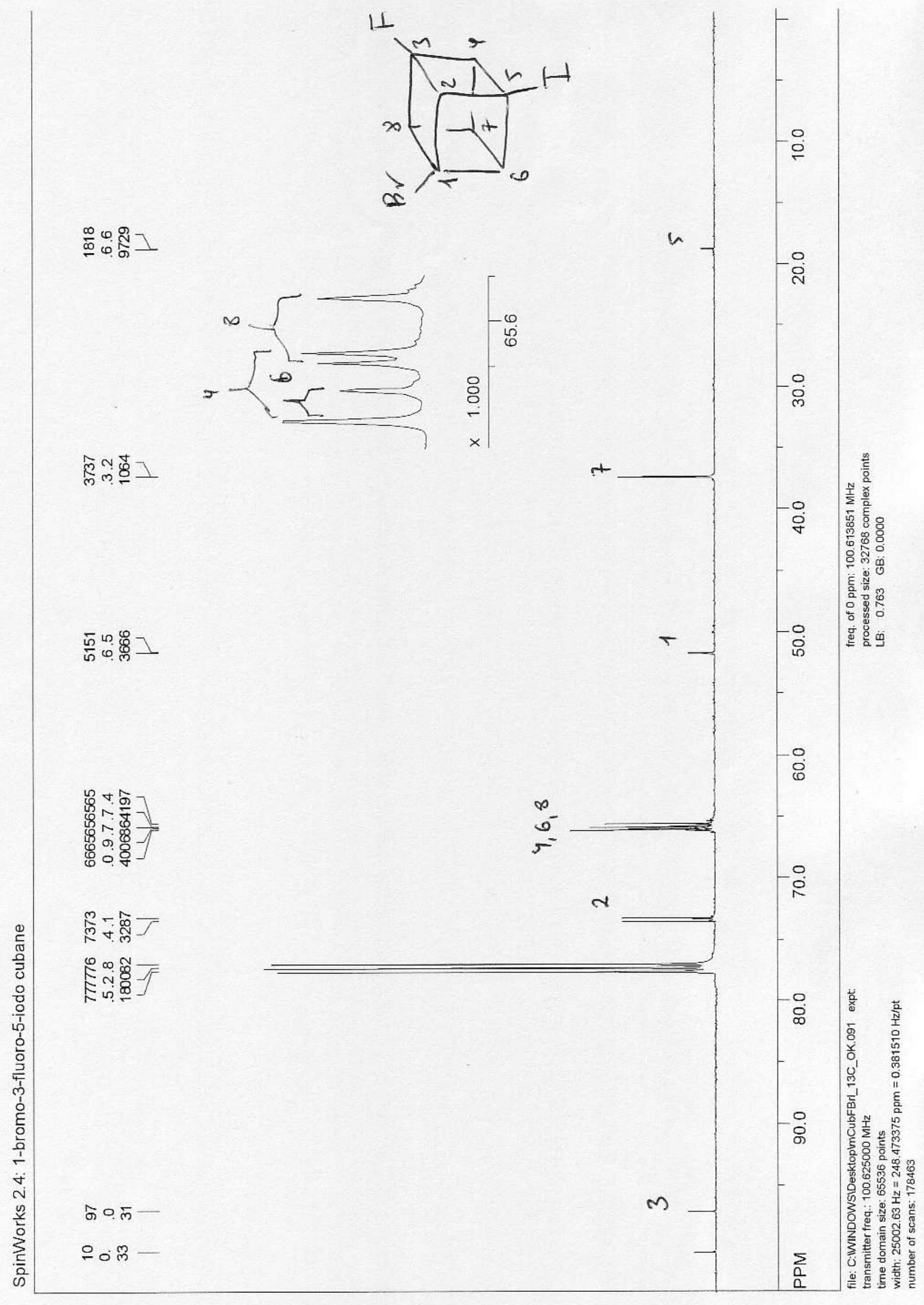




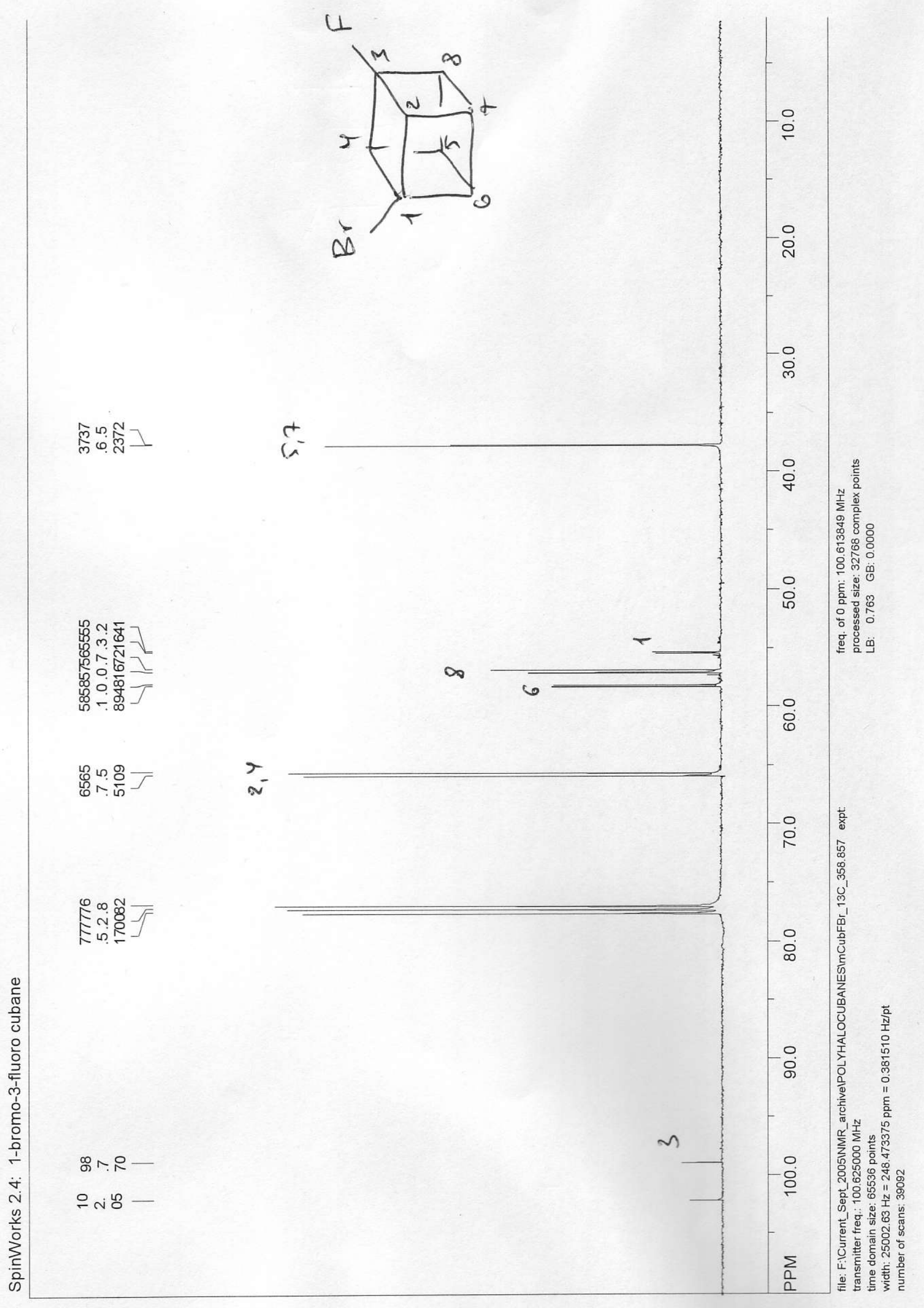




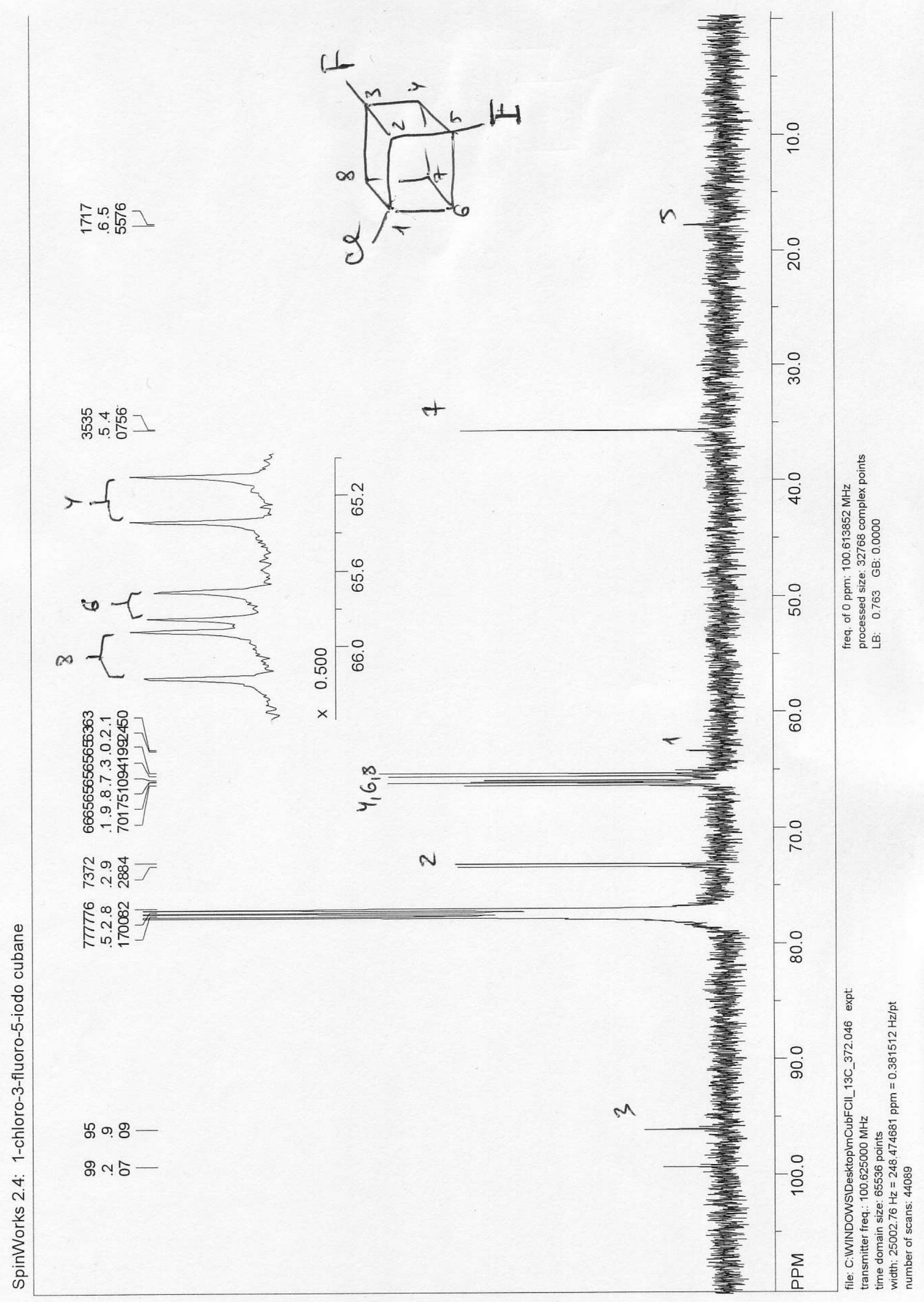




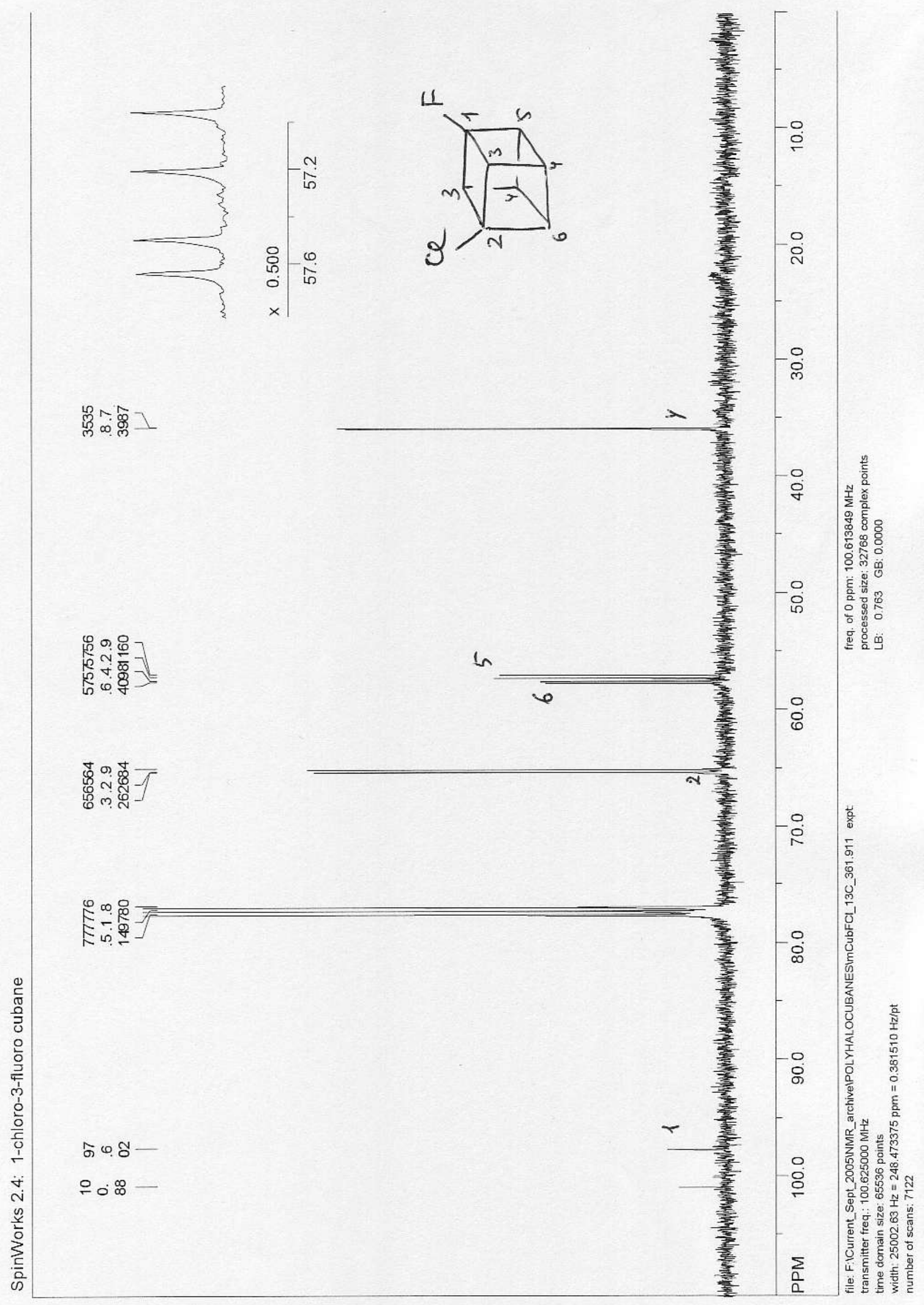

